Meta

Journal des tradlucteurs

Translators' Journal

\title{
Français universel et bon usage
}

\section{Jean-Marie Laurence}

Volume 13, numéro 3, septembre 1968

URI : https://id.erudit.org/iderudit/003417ar

DOI : https://doi.org/10.7202/003417ar

Aller au sommaire du numéro

Éditeur(s)

Les Presses de l'Université de Montréal

ISSN

0026-0452 (imprimé)

1492-1421 (numérique)

Découvrir la revue

Citer cet article

Laurence, J.-M. (1968). Français universel et bon usage. Meta, 13(3), 111-115.

https://doi.org/10.7202/003417ar d'utilisation que vous pouvez consulter en ligne.

https://apropos.erudit.org/fr/usagers/politique-dutilisation/ 


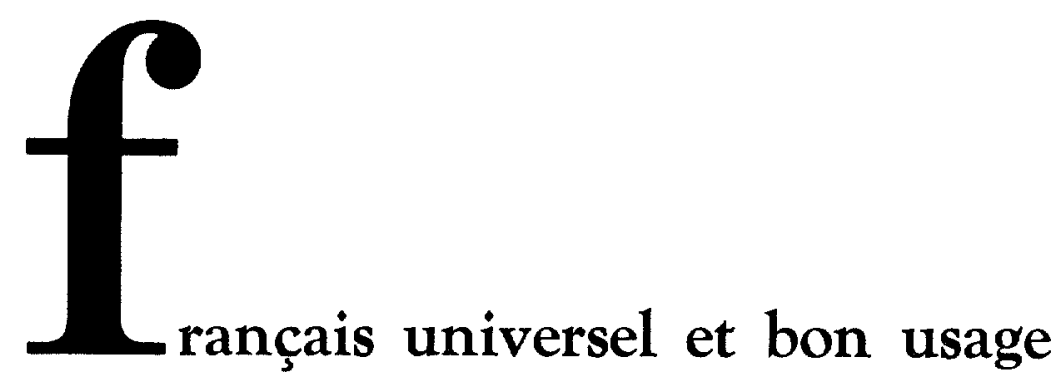

Je ne m'attarderai pas à chercher comment la notion de français universel peut concilier les deux forces contraires qui président à l'évolution des langues et partant des sociétés: la tendance à l'unification et la tendance à la différenciation, dichotomie fondée elle-même sur les antinomies d'ordre philosophique, psychologique, social, politique et linguistique: essence et existence; intelligence et sensibilité; individu et collectivité; internationalisme et nationalisme; langue et parole.

Contentons-nous d'une définition purement empirique et posons en principe que le français universel est l'idiome de la francophonie (c'est-à-dire de la communauté des groupes de langue française). Posons également comme corollaire qu'un idiome de cette nature exige une norme pour exercer pleinement et commodément sa fonction comme instrument de communication. L'histoire de la langue enseigne que cette norme doit assurer l'uniformité presque absolue de la syntaxe et n'admettre les variantes régionales de vocabulaire et de phonétique que dans la mesure où ces variantes n'entravent pas la fonction de communication ni le bon ton (la syntonie sociale) du langage.

Dans la pratique, la norme du français universel, comme de tout idiome, doit trouver son application dans le bon usage.

Or, comment définir le bon usage et quelles en sont les sources ?

Sans passer en revue les définitions de tous les dictionnaires ... et de quelques autres, arrêtons-nous à l'une des définitions de Robert: «Usage. - Utilisation effective (spécialt. normale, correcte) du langage (dans une langue, à une époque donnée, par tous les peuples qui la parlent)." Et Robert cite deux exemples, dont le plus intéressant est sans doute celui de Condillac: "L'usage n'est.pas aussi peu fondé en raison qu'ils le prétendent; il s'établit d'après ce qu'on sent. » D'où l'on peut inférer que, pour Condillac, philosophe sensualiste, le sens linguistique se confond avec la raison raisonnante. Voilà, exprimée sommairement et sans nuances, la doctrine des grammairiens logiciens du XvIII siècle. Cette 
théorie est d'autant plus dommageable que les grammairiens exercent à cette époque un pouvoir presque souverain sur la langue.

Oublions cette doctrine, qui marque un recul par rapport au credo des grands législateurs linguistiques du XvII : Malherbe et Vaugelas. Pour nous en tenir aux Temps Modernes, voilà, semble-t-il, les deux ancêtres de la doctrine linguistique qui se fonde sur l'usage. Malherbe prétend suivre l'usage des crocheteurs du Port-au-Foin, du moins en ce qui touche le vocabulaire; Vaugelas, un peu plus tard, prend pour critère du bon usage la langue de la Cour et de la Ville. Malherbe se fait (théoriquement du moins) l'apôtre de la langue populaire; Vaugelas opte pour le langage aristocratique. Malherbe conduit à Molière; Vaugelas se rattache à Racine.

Si l'on fait abstraction du Xvirre siècle rationaliste et scientifique, on constate que le XIX oscille entre ces deux pôles: le langage populaire d'une part, le langage cultivé d'autre part. Cette oscillation marque la succession des écoles littéraires de l'époque: romantisme, réalisme et naturalisme, symbolisme. Car l'autorité, en matière de langage, est passée des grammariens aux écrivains et aux usagers de la langue.

Du point de vue linguistique, on peut dire que la langue populaire est à la langue cultivée ce que la langue parlée est à la langue écrite.

Or, la langue parlée, en contact immédiat avec la vie courante, exprime plus complètement l'homme que la langue écrite, grâce à l'intonation, aux inflexions ainsi qu'à la mimique et au geste qui accompagnent la parole. Tandis que la langue écrite sert généralement de frein à la langue parlée, elle l'empêche d'évoluer trop vite, elle l'empêche aussi de se trop diversifier. En somme, la langue parlée anime l'évolution linguistique; la langue écrite la dirige et la règle.

Institution sociale à plusieurs égards, la langue obéit aux lois de la sociologie. Or, dans la société, la couche supérieure, qui se reconstitue sans cesse en dépit des changements de régimes et des révolutions, joue le rôle de filtre et de transformateur.

Dans les Temps Modernes, la diffusion sans cesse croissante de l'imprimé et le développement de la littérature ont graduellement et dangereusement réduit l'apport de la langue populaire à l'évolution de l'idiome français. $\grave{A}$ tel point que le bon usage se définit depuis plus d'un siècle: «L'usage des bons écrivains et de la société cultivéc.»C'est proclamer nettement la primauté de la langue écrite, puisque la société cultivée parle "comme les livres» et modèle son langage sur les bons écrivains, c'est-à-dire les écrivains académiques, consacrés par les spécialistes de la critique universitaire.

Trois grands événements sont venus changer tout cela: 1) l'invasion massive de la technique, et singulièrement de la technique américaine; 2) la promotion du prolétariat; 3) la naissance de la radio et de la télévision, instruments de télécommunication.

Conséquence générale: accélération déconcertante du rythme de la vie sur la terre des hommes.

Conséquence linguistique: Littré est devenu précambrien, et tout dictionnaire qui remonte à cinq ans est déjà en partie périmé. Les batifolages d'Abel Hermant vont rejoindre les chinoiseries du Céleste Empire. La linguistique s'annexe la 
psychologie, la psychanalyse, l'ethnographie et la sociologie et commence à lorgner le langage électronique.

En transformant le mode et le style de vie de l'homme du $\mathrm{Xx}^{\mathrm{e}}$ siècle, l'envahissement de la technique, l'industrialisation et la mécanisation agissent nécessairement sur l'évolution de la langue. Le langage paysan est en voie de disparition. La terminologie des anciens métiers semble difficilement adaptable aux techniques nouvelles. Le vocabulaire de la menuiserie, par exemple, passé au malaxage du «gueuloir» populaire, nous déroute presque autant que le lexique d'une langue étrangère. Les vieux mots que voici sont devenus opaques: boutisse, essente, flipot, gobeter, hourdis, linçoir, perré, pureau, soffite, tore, trusquin, volige.

En revanche, les Américains imposent leur terminologie au monde francophone avec les produits de leur technique. L'emprise de cette terminologie s'explique par plusieurs raisons: la force de l'habitude proportionnelle à l'avance de la technique américaine sur certains points et au retard de la traduction française; la supériorité imaginaire, dans la pensée des francophones, de certains termes américains; la valeur expressive réelle de certains vocables particulièrement imagés ou imitatifs; la brièveté de certaines appellations. Ainsi s'établit insensiblement dans notre psychisme le sentiment d'un structuralisme lexical favorable à la terminologie technique américaine. D'où, par recherche des effets d'évocation, les emprunts de la langue courante à la terminologie technique.

La promotion du prolétariat, le développement du syndicalisme et la politisation des masses exercent sans doute sur la langue une influence considérable qu'on n'a pas encore analysée.

Mais les facteurs qui contribuent le plus puissamment à l'évolution linguistique ainsi qu'au rapprochement de la langue écrite et de la langue parlée sont incontestablement la radio et la télévision.

Au Canada français, par exemple, il est indubitable que la radio et la télévision ont largement contribué à la conservation du français en général et à son amélioration dans certains groupes sociaux.

Il est non moins certain que la radio et la télévision sont de véritables écoles de vocabulaire, qu'elles diffusent les mots nouveaux et vulgarisent les termes scientifiques mieux que tout autre moyen. Elles contribuent également à l'uniformisation du français et deviennent les principaux agents de propagation de l'usage, bon ou mauvais. Les plus puissants organismes de réglementation linguistique ne peuvent maintenant méconnaitre ces auxiliaires indispensables.

Ils ne peuvent méconnaître non plus l'influence redoutable de la publicité, qui use et abuse du procédé pédagogique le plus efficace: le texte court indéfiniment répété et ingénieusement illustré.

\section{CONCLUSIONS}

Cet exposé schématique de la situation socio-linguistique du français contemporain autorise, je crois, les conclusions suivantes:

1. Depuis le début des Temps Modernes, le bon usage a obéi, du moins indirectement et dans une mesure variable, à l'autorité des grammairiens en ce 
qui touche la syntaxe et la morphologie et, depuis plus d'un siècle, à l'autorité des lexicologues par la diffusion sans cesse croissante des dictionnaires. On oublie ce fait important quand on affirme que le bon usage est celui des bons écrivains et de la société cultivée.

L'exemple de l'américain, compte tenu d'un laxisme qui nous scandalise, montre cependant que chez nous, grammairiens et lexicologues ont pratiqué un conservatisme paralysant dont nous subissons aujourd'hui les funestes conséquences. Voilà peut-être l'une des causes de l'envahissement du franglais qui nous alarme à juste titre. Le fixisme linguistique est une erreur scientifiquement réfutée depuis longtemps, mais dont l'influence psychologique domine encore la plupart des esprits.

2. Le bon usage français s'est fondé jusqu'à ces derniers temps sur la logique d'une part et sur des critères d'ordre esthétique, littéraire voire mondain d'autre part. Dans son Esthétique de la langue française, Remy de Gourmont est allé jusqu'à soutenir qu'il vaut mieux taire une pensée que de l'exprimer dans une langue qui ne soit pas de pure ascendance latine. C'est de l'aberration.

Il faudrait réviser et reclasser les critères du bon usage à la lumière de la linguistique contemporaine, en s'inspirant davantage du fonctionnalisme et de la psychologie.

3. Dans notre monde en révolution, où l'effervescence des langues accompagne nécessairement le bouillonnement de la vie, le dirigisme linguistique s'impose, à condition qu'il assouplisse ses normes et modifie son rythme.

\section{ASSOUPLISSEMENT DES NORMES}

Les grammairiens français ont beaucoup de mal à s'affranchir de la tradition et des servitudes grammaticales. Ils semblent ignorer l'histoire de la langue, qui les libérerait du fixisme, et la psycholinguistique, qui les éclairerait sur les lois réelles de l'évolution du langage.

La morphologie du verbe, par exemple, présente des irrégularités absolument injustifiables, du point de vue linguistique. En voici quelques exemples. Pourquoi je tressaillirai et je cueillerai? Pourquoi il surseoira et il s'assoira? Pourquoi s'asseoir et je m'assois? Pourquoi vous redites et vous contredisez? Pourquoi des circonflexes qui n'ont plus de valeur phonétique (avant qu'il ết dormi; après qu'il eut dormi) et que les meilleurs écrivains placent à tort et à travers?

Il faudrait aussi mettre fin aux querelles byzantines sur bien que, malgré que; pour autant que; par contre; se rendre compte que; à nouveau, de nouveau; vitupérer, vitupérer contre; invectiver, invectiver contre; d'avance, par avance; c'est à moi à, c'est à moi de, etc. Ces querelles, souvent sans fondement linguistique réel, ne sont bonnes qu'à jeter la confusion dans l'esprit des usagers et à défrayer certaines chroniques de langue dont les auteurs se prononcent le plus souvent au nom du goût (c'est-à-dire leur goût), qu'ils confondent avec l'habitude.

Sans doute, il convient que les linguistes examinent les faits de langage nouveaux avant de les sanctionner; mais cet examen ne doit pas s'éterniser, il doit aboutir à une décision, que les dissidents doivent accepter pour le bien général. 


\section{ACCÉLÉRATION DU RYTHME}

$\mathrm{Au}$ chapitre de l'emprunt, par exemple, pourquoi a-t-il fallu languir des années et vivre dans le péché linguistique avant l'acceptation d'anglicismes aussi indispensables que cumulatif, identifier (quelqu'un), qualifié (au sens de "techniquement compétent $»$ ), exhaustif, constructif (critique constructive), système (dispositif), sophistiqué, suggestion (proposition non officielle), communauté (groupe social), activités (au pluriel), etc.

Il faudrait entreprendre une étude doctrinale de l'emprunt, et particulièrement de l'anglicisme.

4. Enfin, le bon usage du français universel doit se montrer aussi accueillant que possible aux apports du régionalisme. Les pays de la communauté francophone sont sans doute appelés à jouer à l'égard de la langue communautaire un rôle analogue à celui des provinces de France à l'endroit du français officiel.

On sait que la création linguistique est le plus souvent individuelle, même si l'on ne parvient pas toujours à identifier le créateur. De même, les créations régionales, souvent plus concrètes, plus spontanément issues de la vie, peuvent contribuer à enrichir ou à renouveler la langue commune, qui tend fatalement à l'abstraction.

Même à notre époque d'explosion scientifique et technique, il est sage d'écouter ceux qui sont restés plus près de la Terre des Hommes.

Jean-Marie Laurence 